\title{
Endovascular treatment of psuedoaneurysm arising from common hepatic artery bifurcation with complete disruption of gastroduodenal artery and high flow arterioportal fistula
}

\author{
Rinoy R Anand, Mathew Cherian P, Pankaj Mehta, \\ Jenny M Gandhi, Elango S, and Santosh B Patil \\ Department of Neuro and Vascular Interventional Radiology, Kovai Medical Center and Hospital Coimbatore, \\ Tamil Nadu, India

\begin{abstract}
Arterio-portal fistulas (APFs) are characterized by anomalous communication between arteries and the portal vein (PV) system. Treatment of APF is imperative as an emergency or if there is development of portal hypertension/heart failure in chronic cases. Both endovascular and surgical managements can be attempted, however since endovascular management carries comparatively low intra and post procedural morbidity it is mostly preferred. This is a case report on endovascular management of post-traumatic pseudoaneurysm arising from bifurcation of common hepatic artery with complete disruption of the gastroduodenal artery and high-flow APF. This report describes the intraprocedure challenges in exclusion of fistula from the circulation, without disruption of portal system and anticipation of recruitment of new collateral feeders to the fistula immediate post exclusion with its embolization, which needs appropriate positioning of the catheter prior to exclusion of the fistula. (Ann Hepatobiliary Pancreat Surg 2019;23:187-191)
\end{abstract}

Key Words: Psuedoaneurysm; Arterioportal fistula; Endovascular

\section{INTRODUCTION}

Arterio-portal fistulas (APFs) are characterized by anomalous communication between arteries and the portal vein (PV) system. Most of APFs are intrahepatic and secondary to liver cirrhosis, neoplasm, penetrating trauma or congenital vascular malformations. An extrahepatic APF is extremely rare and is almost always attributed to trauma. The clinical presentation may vary from an asymptomatic individual to patients with severe portal hypertension, gastrointestinal bleeding, refractory ascites and hepatic encephalopathy. ${ }^{1}$

Since the prognosis of APF is associated with potential life threatening complications, an early and effective treatment is critical. Surgical and endovascular treatment approaches are available, however endovascular management is superior due to its less invasiveness and relatively early patient rehabilitation. ${ }^{2}$
This is a case report on endovascular management of post-traumatic pseudoaneurysm arising from bifurcation of common hepatic artery with complete disruption of the gastroduodenal artery and high-flow APF. Although there are endovascular management of high-flow APFs described in literature, this is the first case report of endovascular treatment of avulsion of the gastroduodenal artery origin with high-flow APF with challenges in management for this case. An institutional review board waiver was granted for preparation of this report.

\section{CASE}

A 57-year-old male was admitted with alleged history of road traffic accident (blunt trauma to abdomen on the same day) and acute onset abdominal pain. On examination he was conscious, oriented and hemodynamically stable with BP of 130/80 mmh and heart rate of 98/min.

Received: October 11, 2018; Revised: February 1, 2019; Accepted: February 7, 2019

Comesponding author: Mathew Cherian $\mathrm{P}$

Department of Neuro and Vascular Interventional Radiology, Kovai Medical Center and Hospital Coimbatore, Avanashi Road, Coimbatore, Tamil Nadu 641014, India

Tel: +914224323800, Fax: +91422827782, E-mail: dr.mathewcherian@gmail.com

Copyright (C) 2019 by The Korean Association of Hepato-Biliary-Pancreatic Surgery

This is an Open Access article distributed under the terms of the Creative Commons Attribution Non-Commercial License (http://creativecommons.org/ censes/by-nc/4.0) which permits unrestricted non-commercial use, distribution, and reproduction in any medium, provided the original work is properly cited. Annals of Hepato-Biliary-Pancreatic Surgery • pISSN: 2508-5778 - elSSN: 2508-5859 

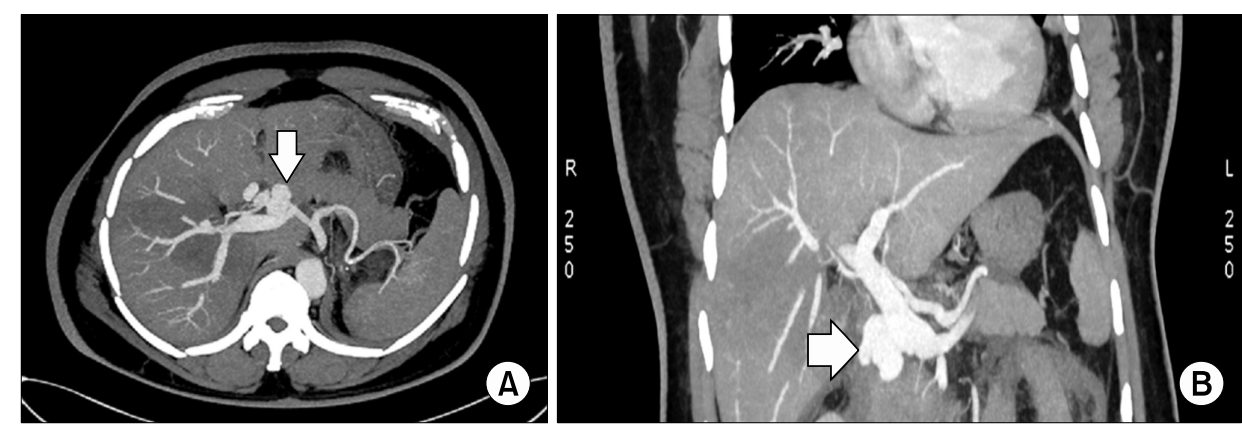

Fig. 1. Computed tomography (CT) images: Axial (A) and coronal (B) section in arterial phase of contrast CT scan showing pseudoaneurysm (white arrow) anterior to the proximal main portal vein with early filling of the entire portal system.

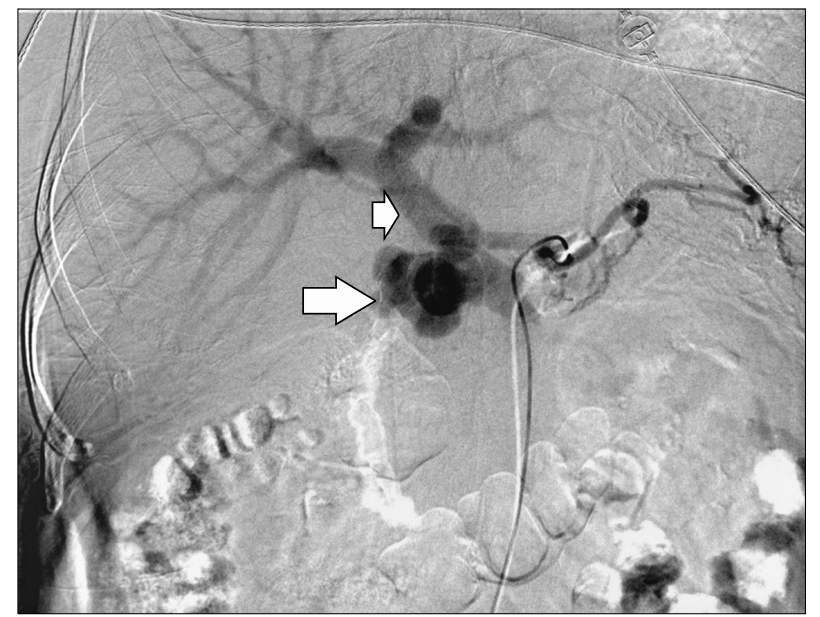

Fig. 2. Celiac angiogram demonstrating pseudoaneurysm (big arrow) at the common hepatic artery bifurcation and filling of portal vein (small arrow).

Abdominal examination revealed right hypochondriac tenderness. Abdomen was soft without any evidence of guarding rigidity.

A triple phase contrast enhanced CECT scan of abdomen was done, using Somatom Force Siemens CT (Erlangen, Germany). It revealed AAST grade IV liver injury with a pseudoaneurysm (Fig. 1) arising from the junction of common hepatic artery with non-visualization of gastroduodenal artery (GDA). There was early filling of main portal vein and its branches in arterial phase at the level of pseudoaneurysm, consistent with APF.

He was taken up for catheter angiogram. Through right common femoral artery access (short sheath, 4F) a celiac artery angiogram was done, which revealed a pseudoaneurysm $(4.2 \times 2.9 \mathrm{~cm})$ arising from bifurcation of common hepatic artery with high-flow APF and non visualization of gastroduodenal artery (Fig. 2). There was faint opacification of proper hepatic artery. Superior mesenteric artery (SMA) angiogram revealed retrograde filling of gastroduodenal artery (GDA) via pancreatico-duodenal arcade



Fig. 3. Superior mesenteric artery angiogram showing filling of pseudoaneurysm (big arrow) through inferior pancreaticoduodenal collateral. Filling of main portal vein (small arrow) can also be seen.

with filling of APF (Fig. 3). Hence a second access was taken at the right common femoral artery and a SIM 1 catheter was placed in the SMA for guidance.

Right CFA 4F short sheath was exchanged with $5 \mathrm{~F}$ long sheath. Progreat microcatheter with micro-wire (Terumo, Shibuya, Japan) was negotiated into proper hepatic artery distal to fistula. It was further advanced deeply into the branch of right hepatic artery. Progreat wire was exchanged with coronary wire and kept in position.

A third access was taken in the left CFA and a 7F long sheath was positioned in the common hepatic artery. Through this a Bernstein catheter was navigated into proximal gastro duodenal artery. Under the guidance of multiple injections from SMA a Progreat microcatheter with micro-wire was traversed across the rent in GDA. The micro-wire was exchanged with a Whisper extra support wire (Abbott, Illinois, USA) and a $4.2 \times 29 \mathrm{~mm}$ balloon expandable stent graft (Graftmaster, coronary stent graft, Abbott, Illinois, U.S.A) was deployed in GDA. 
Direct embolization of this segment was not attempted as there was high risk of migration of the embolizing agent (eg: Coils) to portal vein system through the high-flow fistula. Post-deployment angiography showed good continuity between the hepatic artery and the distal GDA. However, there was persistent opacification of the pseudoaneurysm and portal vein from the junction of stent graft in GDA and common hepatic artery (Fig. 4). The stent graft could not be positioned proximally, since it would occlude the common hepatic artery and its bifurcation. The balloon catheter was then replaced with Progreat microcatheter which was placed distal to the stent graft. This was primarily done to ensure that the distal access of the vessel was available at later stage of treatment.

Following this a diagnostic catheter was tracked over the coronary wire in the distal hepatic artery branch with-

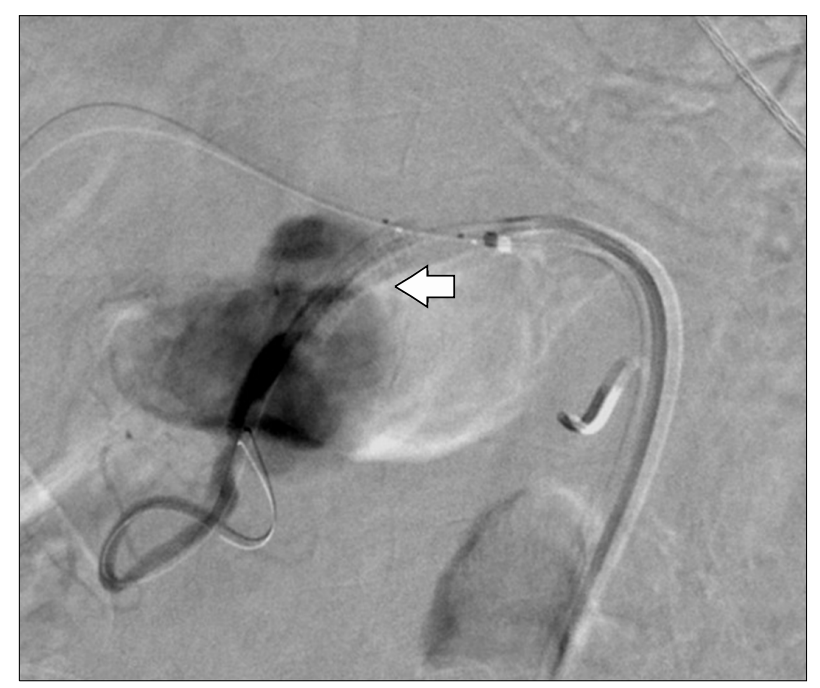

Fig. 4. Post stent graft deployment in gastro duodenal artery (stent-white arrow). Angiogram from common hepatic artery revealing residual filling of pseudoaneurysm. in the $5 \mathrm{~F}$ sheath and the coronary wire was replaced with extrastiff wire and $5 \mathrm{~F}$ sheath was replaced with $9 \mathrm{~F}$ sheath. After ascertaining right position, a $10 \mathrm{~mm} \times 40 \mathrm{~mm}$ self-expanding stent graft (Fluency, BARD, Arizona, USA) was deployed across the rent from proper hepatic artery to common hepatic artery. Post procedure celiac angiography showed good filling of the hepatic artery and its branches without filling of the pseudo aneurysm (Fig. 5).

SMA check injection at this point through SIM 1 catheter showed minimal filling of pseudoaneurysm though a leak around the previously placed stent graft in GDA (Fig. 6A). Since the Progreat catheter was pre emptively positioned in distal GDA before deploying the Fluency stent graft, it was easy to deploy Nester coils (Cook medical, Bloomington, USA) and thus embolization of the GDA distal to stent graft was done using $125 \mathrm{~cm}, 0.018$ " pushable fibered Nester coils (Fig. 6B).

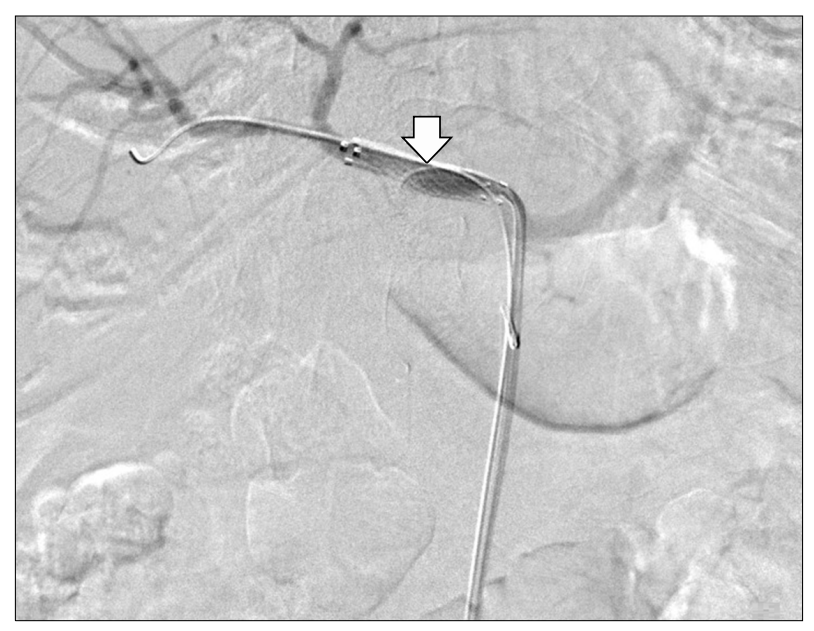

Fig. 5. Post stent graft deployment in common hepatic artery (stent graft in common hepatic artery-white arrow). Celiac angiogram shows no residual filling of the pseudoaneurysm from celiac branches.
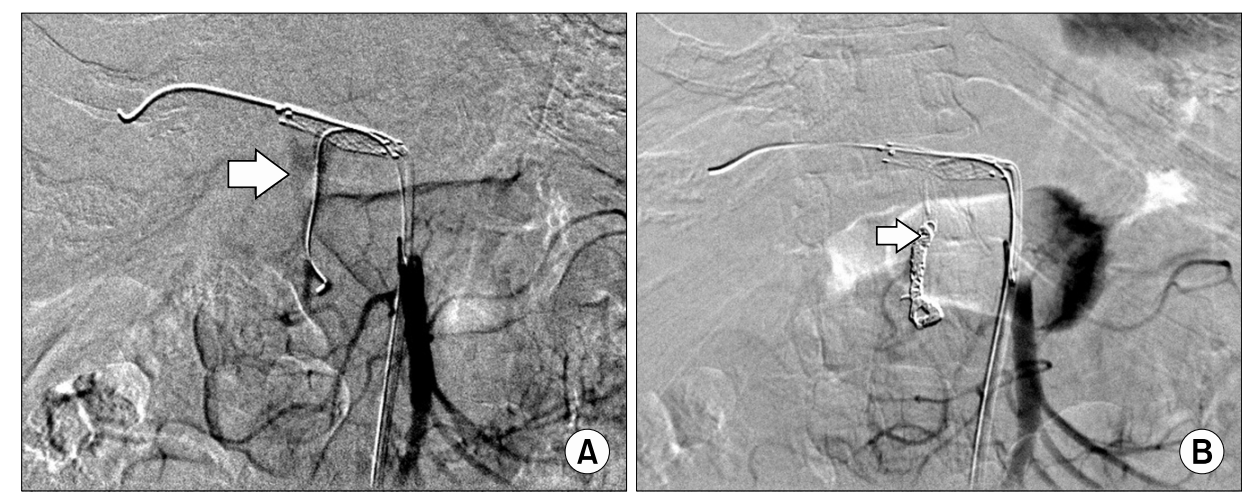

Fig. 6. SMA angiogram: (A) Post stent deployment superior mesenteric artery (SMA) injection revealing residual filling (white arrow) of the pseudoaneurysm through collateral from pancretico duodenal arcade; (B) Post coiling status of gastroduodenal artery with angiogram from SMA showing no residual filling of pseudoaneurysm. 

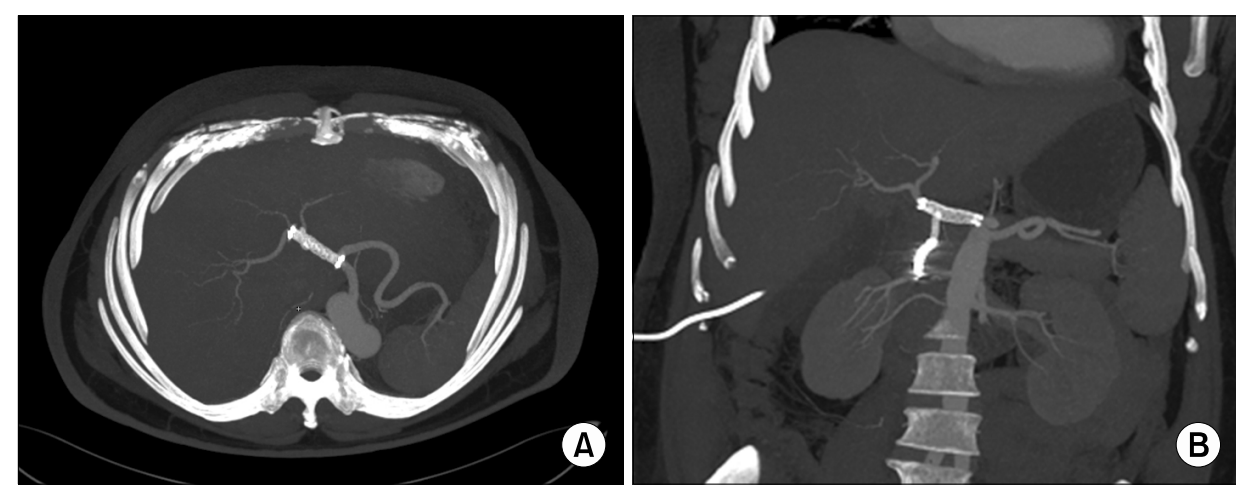

Fig. 7. Computed tomography (CT) images: Axial (A) and coronal (B) maximum intensity projection (MIP) sections of follow-up CT (arterial phase) showing stent in common hepatic artery without residual filling of pseudoaneurysm or arterio-portal fistula. Normal filling of branches of common hepatic artery and coils in gastroduodenal artery (B) are also seen.

Post procedure angiogram at celiac artery and SMA showed complete obliteration of pseudoaneurysm without residual filling of the portal vein.

Patient was hemodynamically stable and was monitored with complete blood count and liver function test. Following one week of trauma, the patient developed subcapsular collection in segment VI of liver for which pigtail drainage catheter was positioned.

At 15 days post-intervention, the patient developed gradual rise in serum bilirubin with elevated direct bilirubin. Total bilirubin was $9.88 \mathrm{mg} / \mathrm{dl}$ with direct bilirubin value of $7.46 \mathrm{mg} / \mathrm{dl}$. CECT abdomen was performed to rule out etiology of hyperbilirubinemia. It revealed complete thrombosis of pseudoaneurysm sac with normal filling of portal vein. Hepatic artery (Fig. 7), portal vein and its branches showed normal contrast opacification. There was minimal dilatation of central IHBR with proximal and mid CBD upto the level of thrombosed psuedoanuerysm sac beyond which $\mathrm{CBD}$ was not prominent which suggested mass effect and compression on CBD by thrombosed pseudoaneurysm sac. Gastroenterologist opinion was seeked and patient successfully underwent endoscopic CBD stenting. Patient was followed up for 2 months after stenting. His bilirubin levels showed a decreasing trend and normalization.

\section{DISCUSSION}

APFs are rare vascular disorders that can occur extraor intra-hepatically. Etiology of hepatic APF includes congenital vascular malformation, cirrhosis, malignancy and trauma. ${ }^{3}$ Most of these causes result in intrahepatic APF. Traumatic cause for an APF is mostly related to surgical interventions or liver biopsy. Non-iatrogenic abdominal trauma that is related to APF is rarely reported. ${ }^{4}$ In our case the patient had history of blunt trauma to the abdomen which resulted in avulsion of common hepatic artery bifurcation with development of pseudoaneurysm and high-flow APF.

Hepatic arterial blood flow into the portal system causes venous congestion and back flow in portal vein. This can culminate as portal hypertension and severe mesenteric venous congestion. As pressure changes affect intima of veins, the absorption of water and sodium from colonic mucosa is reduced, causing frequent watery diarrhea. ${ }^{3,5}$ Other complications such as variceal bleeding and refractory ascites can also occur secondary to portal hypertension.

The clinical spectrum of presentation ranges from symptom-free individuals to patients with severe portal hypertension. ${ }^{6}$ One of the most common presentations of an acute APF is gastrointestinal bleed following an iatrogenic or accidental trauma. ${ }^{5,7}$ The presentation of hepatic APFs depends on its etiology, size, and flow through the shunt and thereby its hemodynamic consequences. ${ }^{5}$ In contrast to systemic APF, the occurrence of congestive cardiac failure is comparatively less in hepatic APF due to resistance offered by hepatic sinusoids. ${ }^{8}$

The rationale behind treatment of an asymptomatic APF is to prevent the development of portal hypertension and its complications. ${ }^{5}$ While the majority of patients have been treated surgically in the past, endovascular interventional radiological procedures are being successfully performed with increasing frequency recently due to lesser invasiveness, ${ }^{9}$ decreased morbidity, better safety, better results and lesser hospital stay. ${ }^{3,5,6}$ Although surgical procedures can reduce portal venous pressure and obliterate the fistula, increase in operative time and morbidities in post- 
operative period makes this approach undesirable. ${ }^{3}$ Currently surgical management is reserved mostly when endovascular interventional treatment fails.,

Although endovascular management of high-flow APFs has been described in literature, this is the first case report of endovascular treatment of pseudoaneurysm from common hepatic artery bifurcation and avulsion of the gastroduodenal artery origin with high-flow APF. There were challenges in the management of this case. The initial challenge was to cross the disrupted segment and to successfully deploy the stent graft across the rent in GDA. The stent graft was mandatory since the second challenge in this case was the large pseudoaneurysm directly communicating to portal vein. Placing coils in the pseudoaneurysm can cause its migration into portal vein with resultant thrombosis of the portal vein which could be fatal. This case describes the importance of anticipating problems before hand. Had the microcatheter not been placed across the stent graft in the GDA, the procedure would have been useless or extremely difficult to complete because crossing a stent graft across the rent would have been nearly impossible.

The primary aim of any interventional procedure in an aorto portal fistula is to achieve selective fistula closure and preservation of adjacent normal vasculature. In case of large APF the development of vascular steals through collateral circulation must also be adequately controlled for optimum treatment and prevention of residual or recurrent filling of the APF. ${ }^{5}$ In our case we successfully covered the entry point of fistula using stent graft and embolized the collaterals using coils, resulting in complete obliteration of fistula and thrombosis of the pseudoaneurysm.
In conclusion, endovascular technique can be considered as less invasive and first line treatment option in APF. Obliteration of inflow and precluding collateral feeders can improve treatment results.

\section{REFERENCES}

1. Hirakawa M, Nishie A, Asayama Y, Ishigami K, Ushijima Y, Fujita N, et al. Clinical outcomes of symptomatic arterioportal fistulas after transcatheter arterial embolization. World J Radiol 2013;5:33-40.

2. Khosla A, White B, Kalva S, Pillai A. Endovascular management of a post-traumatic complex arterioportal fistula. J Vasc Med Surg 2015. doi: 10.4172/2329-6925.1000198.

3. Zhang WG, Li Z, Ding PX, Ren JZ, Ma J, Zhou PL, et al. Endovascular treatment of an unusual primary arterioportal fistula complicated by cavernous transformation of the portal vein caused by portal thrombosis. Ann Vasc Surg 2014;28:491. e5-e8.

4. Han P, Yang L, Huang XW, Zhu XQ, Chen L, Wang N, et al. A traumatic hepatic artery pseudoaneurysm and arterioportal fistula, with severe diarrhea as the first symptom: a case report and review of the literature. Medicine (Baltimore) 2018;97: e9893.

5. Kumar A, Ahuja CK, Vyas S, Kalra N, Khandelwal N, Chawla $\mathrm{Y}$, et al. Hepatic arteriovenous fistulae: role of interventional radiology. Dig Dis Sci 2012;57:2703-2712.

6. Tomczak R, Helmberger T, Görich J, Schütz A, Merkle E, Brambs HJ, et al. [Abdominal arteriovenous and arterio-portal fistulas: etiology, diagnosis, therapeutic possibilities]. Z Gastroenterol 1997;35:555-562. German.

7. Vauthey JN, Tomczak RJ, Helmberger T, Gertsch P, Forsmark $\mathrm{C}$, Caridi J, et al. The arterioportal fistula syndrome: clinicopathologic features, diagnosis, and therapy. Gastroenterology 1997;113:1390-1401.

8. Guzman EA, McCahill LE, Rogers FB. Arterioportal fistulas: introduction of a novel classification with therapeutic implications. J Gastrointest Surg 2006;10:543-550.

9. Bouziane Z, Ghissassi B, Bouayad M, Sefiani Y, Lekehal B, El Mesnaoui A, et al. Successful endovascular management of postoperative arterio portal fistula. Ann Vasc Surg 2011;25: 385.e1-e3. 